NO.10 Journal of Petroleum Research \& Studies ( JPR \& S)

\title{
Borehole Stability in Shale Formation: Modeling the Effects of Molecular Weight and Concentration of Polymers in the Drilling Fluids Formulation
}

\author{
Khoshniyat A. ; Shojaei M. ; Jarahian K. ; Mirali M*; Ghorashi S. "; Aalaie.J. *; \\ Moalemi H. ${ }^{* *}$ \\ * Polymer Science and Technology Division, Research Institute of Petroleum \\ Industry (RIPI), \\ ** Iranian Central Oil Company
}

\section{$\underline{\text { Abstract }}$}

A new experimental model was developed to predict the role of special polymeric additives, in the drilling fluid formulation, on the wellbore stability in shale formation. The shale formation was regarded as a non-ideal membrane and the effects of various characteristics of the added polymers were studied on the membrane reflection coefficient. The model was applied to unique field data from the oil field in south of Iran, including clay structure, cation exchange capacity (CEC), density and porosity of the shale. The results, using various polyglycols and polyacrylamides as the polymeric additive, showed that the structure of the polymeric chains e.g. type and content of ionic segments had significant effect on their adsorption mechanism and its strength. It was concluded that increasing the molecular weight of the polymer chains decreased the rate and amount of the adsorption due to the increasing of the entanglements between the chains which in turn limited their mobility. So, adsorption of the polymeric material on the shale had significant impress on its performance as a membrane by increasing the shale reflection coefficient enhancing its stability during drilling process. Finally, the developed model results were in good agreement by experimental test results which was done in a specific shale stability set up. 


\section{NO.10 Journal of Petroleum Research \& Studies ( JPR \& S)}

\section{Introduction}

Replacement of oil-based muds (OBMS) with water-based muds (WBMs) has been the subject of many researches in the drilling industry for the last decades. Recent progresses in the understanding of different types of drilling muds have identified that WBMs are highly desirable due to their great properties, e.g. lower cost, strong shale-stabilizing effect and less negative impacts on the environment. The mechanisms of shale-stabilizing properties of WBMS have been studied by other researchers [1-5]. It has been found that shale systems can be considered as semi permeable membrane which act selective to the transport of water and solutes (ions) and thereby may act as non-ideal membranes [6]. The flows of solvent (water in the case of using water-based muds) and solute (salt) through the shale can be described by the following phenomenological equations under isothermal condition:

$$
\begin{aligned}
& J_{v}=L_{p} \Delta P+L_{p d} \Delta \pi \\
& J_{d}=L_{d p} \Delta P+L_{d} \Delta \pi
\end{aligned}
$$

Where $J_{v}$, is the volumetric flow (the total flow of water and salt) through the shale membrane, $\mathrm{J}_{\mathrm{d}}$ is the diffusion flow which is a measure of relative velocity of salt flux to water flux, $\Delta \mathrm{P}$ is hydrostatic pressure differender is the osmotic pressure resulting from chemical potential differences between the shale and the drilling fluids which cause the osmotic backflow of pore water into or from the drilling mud depends on the water activity of the fluid of them [7], $L_{p}$ is hydraulic permeability coefficient which is related to the conventional permeability coefficient, $\mathrm{L}_{\mathrm{d}}$ is solute diffusional mobility coefficient, $\mathrm{L}_{\mathrm{pd}}$ is osmotic flow coefficient and $\mathrm{L}_{\mathrm{dp}}$ is ultrafilltration coefficient. According to Onsagar's reciprocal law, the coefficients $\mathrm{L}_{\mathrm{pd}}$ and $\mathrm{L}_{\mathrm{dp}}$ are identical. Therefore, only three independent coefficients are necessary to describe the current flow system. Under equilibrium 
NO.10 Journal of Petroleum Research \& Studies ( JPR \& S)

condition, the volumetric flux in equation (1) is zero and the following expression can be obtained:

$$
\frac{\Delta P}{\Delta \pi}=-\frac{L_{p d}}{L_{p}}=\sigma
$$

Where $\sigma$ is the membrane reflection coefficient and $\Delta \pi$ is defined by:

$\Delta \pi=\frac{\mathrm{RT}}{\mathrm{V}_{\mathrm{w}}} \ln \left[\frac{\mathrm{a}_{\mathrm{w}}^{\mathrm{sh}}}{\mathrm{a}_{\mathrm{w}}^{\mathrm{df}}}\right]$

Where $\mathrm{T}$ is temperature $\left({ }^{\mathrm{o}} \mathrm{K}\right), \mathrm{R}$ is the gas constant $\left(\mathrm{J} \mathrm{mol}^{-1} \mathrm{~K}^{-1}\right), \mathrm{V}_{\mathrm{W}}$ is the partial molar volume of water $\left(\mathrm{m}^{3} \mathrm{~mol}^{-1}\right), \mathrm{a}_{\mathrm{w}}^{\mathrm{sh}}$ and $\mathrm{a}_{\mathrm{w}}^{\mathrm{df}}$ are the water activities of the shale pore fluid and the drilling fluid respectively. An ideal semi-permeable membrane is characterized by $\sigma=1$, indicating that there is no solute flux through the membrane. With leaky membranes like shale, the reflection coefficient will be between zero and one $(0<\sigma<1)$. For semi permeable membrane, membrane reflection coefficient, $\sigma$, is defined by $[8,9]$ :

$$
\sigma=1-\frac{k_{s}\left(R_{c a-w}+1\right)}{\left\{\left[R_{c a}-w\left(\frac{c_{a}}{c_{c}}\right)+1\right]+R_{a-m w}\left[R_{c a-m}\left(\frac{c_{a}}{c_{c}}\right)+1\right]\right\} \phi_{w}}
$$

Where $\mathrm{K}_{\mathrm{s}}=\mathrm{Ca} / \mathrm{Cs}$. Ca is the anion concentration $\left(\frac{\mathrm{mol}}{\mathrm{cm}^{3}}\right)$ within the membrane pores which can be calculated by the following equation:

$$
c_{a}=\frac{-1}{2} E \rho\left(1-\phi_{w}\right)+\frac{1}{2}\left[E^{2} \rho^{2}\left(1-\phi_{w}\right)^{2}+4 c_{s}{ }^{2}{\phi_{w}}^{2}\right]^{\frac{1}{2}}
$$

Where $\mathrm{E}$ is the cation exchange capacity (CEC) of the shale $(\mathrm{meq} / \mathrm{g}), \rho$ is density of shale $\left(\mathrm{g} / \mathrm{cm}^{3}\right)$ and $\varphi_{\mathrm{w}}$ are the dry density and the porosity of the shale respectively. $\mathrm{C}_{\mathrm{C}}$ in the above equation is the concentration of cation $\left(\frac{\mathrm{mol}}{\mathrm{cm}^{3}}\right)$ within the membrane pores which is given by:

$$
c_{c}=c_{a}+E \rho\left(1-\phi_{w}\right)
$$

The $\mathrm{R}$ terms in the above equation are ratios of frictional coefficients. 


\section{NO.10 Journal of Petroleum Research \& Studies ( JPR \& S)}

The main objective of the present work was to study the effect of different polymers on the reflection coefficient of shale of oil field in Iran and therefore its stability. It was tried to predict the dispersion state of the stabilization of the shale via the variation of reflection coefficient parameter. Also, a mathematical relation was derived between the properties of used polymers and this parameter.

\section{Experimental}

\subsection{Materials}

The polyacrylamide used in this study was PHPA, obtained from SNF. The polyethylene glycol, PG, was obtained from Aldrich. Shale samples were taken out from the oil field in south of Iran. $\mathrm{NaOH}, \mathrm{HCl}$ and Phenol phetalein as detector were obtained from Merck for determination of CEC parameter.

\subsection{Preparation of samples}

All the samples were prepared in ambient temperature by solving the different weights of different polymers in water which was contained specific concentration of shale with mesh of 18 using stirring by magnet and hot roll. The stirring process was continued up to 16 hours. Obtained samples were filtered and dry polymers treated shale was obtained for next tests.

\subsection{CEC measurement}

CEC measurement was carried out using titration of acid-base. $\mathrm{HCl}$ and $\mathrm{NaOH}$ were used for this test. Test medium was containing $0.6 \mathrm{gr} \mathrm{NaOH}$ and 1 gr polymer treated shale. After 16 hours, addition of detector and acid to the test medium was started. Addition of acid was continued until the color of test medium changed from violet to white. At this point, the volume of acid was recorded and CEC was obtained. 
NO.10 Journal of Petroleum Research \& Studies ( JPR \& S)

\subsection{Density measurement}

Density of different polymer treated samples was derived by division of their mass to their volume.

\subsection{Porosity measurement}

Porosity of different samples was derived by measurement of the flow rate of nitrogen trough the samples.

\section{Results and discussion}

Using various polymers in the water based drilling fluid formulation is essential for shale stabilization during the drilling process. However, equation 5 does not consider the effects of polymer parameters on the reflection coefficient. The aim of this research work to modify the $\mathrm{C}_{\mathrm{a}}$ in equation 5 not only depends on the CEC, density and porosity parameters of the shale, but also on the concentration $\left(\mathrm{C}_{\mathrm{p}}\right)$ and molecular weight $\left(\mathrm{M}_{\mathrm{w}}\right)$ of the used polymers.

At first, we utilized shale-outcropped samples from one of the south western Iranian oil fields. Therefore samples were exposed to water-based drilling mud (WBM) containing partially hydrolyzed polyacrylamide (PHPA) with different molecular weights and polyglycol, and then mixed with two kinds of methods, e.g. ball mill and stirrer. So the constant values of equation 5 were determined, subsequently shale reflection coefficient (SRC) was calculated in table 1 . According to these results, increase of SRC by polymeric additives was confirmed. Experiments showed that 12 million Dalton polymer molecular weights was the most optimum $\mathrm{M}_{\mathrm{W}}$ for improving of SRC. It can be explained by physico-chemical configuration of polymeric chains and ability of that polymer in better adsorption on shale sample's surfaces.

Buckingham dimensional analysis method was used in order to develop equation 5 for polymer containing systems. At first dimensionless parameters $\left(\pi_{1}, \pi_{2}, \pi_{3}\right.$ and $\pi_{4}$ ) were defined by considering the dimensions of the different parameters such as 


\section{NO.10 Journal of Petroleum Research \& Studies (JPR \& S)}

$\mathrm{C}_{\mathrm{a}}, \mathrm{CEC}, \rho, \mathrm{C}_{\mathrm{p}}, \mathrm{M}_{\mathrm{w}}, \varphi_{\mathrm{w}}$ and two independent parameters (Mass and length) in the following way:

$$
\begin{aligned}
& \pi_{1}=\frac{C_{a} \cdot M_{w}}{C_{p} \cdot C E C} \quad \text { (8) } \quad \pi_{2}=\frac{\rho \cdot M_{w}}{C_{p} \cdot C E C} \quad \text { (9) } \quad \pi_{3}=\frac{\varphi_{w} \cdot C_{p} \cdot C E C}{M_{w}} \\
& \pi_{4}=\frac{M_{w}}{C E C}
\end{aligned}
$$

Then power law relation was derived between these parameters:

$$
\Rightarrow \frac{C_{a} \cdot M_{w}}{C_{p} \cdot C E C}=\alpha\left(\frac{\rho \cdot \varphi_{w} \cdot M_{w}}{C E C}\right)^{\beta}
$$

By plotting the left hand side of the above relation against its right hand side, using the data of Table 1 for the systems containing various concentrations of different polymers, the parameters $\alpha$ and $\beta$ were calculated as 2875.9 and 0.9741 respectively. Consequently, $\mathrm{C}_{\mathrm{a}}$ can be written as follows:

$\Rightarrow C_{a}=C_{p} \times 2875.9(\rho . \varphi)^{0.9741}\left(\frac{C E C}{M_{w}}\right)^{1.9741}$

Using the obtained $\mathrm{C}_{\mathrm{a}}$ in equation 13 , one would be able to predict the performance of drilling fluids in the presence of polymeric materials in their formulation.

\section{Shale stability investigation in set up}

In this section, core samples prepared in 2 inch diameter and 1 inch height were set inside the core holder after initial saturation with water formation. Then core sample was exposed to two pore fluid (formation water) and drilling fluid with constant pressure of 200 and 220 bar respectively and 220 bar as an overburden pressure. Due to drilling fluid penetration and also osmosis pressure fluctuation was monitored in pore fluid section and this pressure was considered as a determining factor for shale stabilization. 


\section{NO.10 Journal of Petroleum Research \& Studies ( JPR \& S)}

Figures 1 and 2 depict the result of this experiment. As it was shown, oil-based mud (OBM) has no penetration in shale samples and doesn't cause any change in pore pressure vessel. This phenomenon means that the shale stability has been guaranteed. On the other hand in the presence of water based drilling mud (WBM) containing polymeric components, osmosis phenomenon can be predominant mechanism of shale stabilizer. At the moment of water exposition to shale sample, water started to penetrate into samples and therefore pressure changes in pore vessel can be observed. So after some fluctuation, this event reaches to equilibrium state.

In this research, reflection coefficient can be calculated by equation 3 . The LENNTECH software was used for calculate the $\Delta \pi$. The Osmosis pressure was calculated 152.7 bar by interring the concentration of anions and cations existing of formation and drilling fluids and also TDS (total dissolved solids) value (table 2) in mentioned software. On the other hand, $\Delta \mathrm{P}$ (the difference pressure between formation and drilling fluids of core sample) was observed 131 bar, through figure (2).

The results of experiments show which the difference between reflection coefficients obtained from setup system and modified model was $12 \%$. It can be attributed to other parameters including temperature and pressure.

\section{Conclusion}

The impact of different polymers on the stability of shale of Iran oil field has been studied. The effects of density, porosity and cation exchange capacity (CEC) of the shale were studied by means of a mathematical model, based on Buckingham theory. Finally, a new mathematical equation for reflection coefficient was obtained which included polymeric parameters such as molecular weight and concentration. Difference between reflection coefficients obtained from experimental setup system and modified model was $12 \%$. It can be attributed to 


\section{NO.10 Journal of Petroleum Research \& Studies ( JPR \& S)}

other parameters including temperature and pressure on the amount of shale reflection coefficients.

Table (1) Reflection coefficients calculated for shale samples

\begin{tabular}{|c|c|c|c|c|c|c|c|c|c|}
\hline Shale Sample & PAB 11 & PAB11-8m & PAB11-8b & PAB11-12m & PAB11-12b & $\begin{array}{l}\text { PAB } \\
11-16 \mathrm{~m}\end{array}$ & PAB11-16b & PAB11-P50m & $\begin{array}{l}\text { PAB11- } \\
\text { P50b }\end{array}$ \\
\hline $\begin{array}{l}\text { density } \\
(\mathrm{g} / \mathrm{cm} 3)\end{array}$ & 1.34 & 1.29 & 0.87 & 1.30 & 1.75 & 0.87 & 1.04 & 1.59 & 1.59 \\
\hline prosity (\%) & 2.01 & 3.69 & 2.63 & 6.30 & 9.94 & 2.79 & 4.06 & 6.20 & 5.64 \\
\hline $\begin{array}{l}\mathrm{Ca} \\
(\mathrm{mol} / \mathrm{cm} 3)\end{array}$ & 1.65526103 & 2.43472098 & 1.51363811 & 6.08662636 & 12.37473149 & 1.48188801 & 4.00204083 & 6.85341008 & 7.01755881 \\
\hline $\begin{array}{l}\text { Cs } \\
(\mathrm{mol} / \mathrm{cm} 3)\end{array}$ & 0.3270000 & 0.3270000 & 0.3270000 & 0.3270000 & 0.3270000 & 0.3270000 & 0.3270000 & 0.3270000 & 0.3270000 \\
\hline Ks & 5.061960 & 7.445630 & 4.628863 & 18.613536 & 37.843216 & 4.531768 & 12.238657 & 20.958441 & 21.460424 \\
\hline R ca-w & 1.63 & 1.63 & 1.63 & 1.63 & 1.63 & 1.63 & 1.63 & 1.63 & 1.63 \\
\hline R ca-m & 1.08 & 1.08 & 1.08 & 1.08 & 1.08 & 1.08 & 1.08 & 1.08 & 1.08 \\
\hline $\begin{array}{l}\text { CEC (meq/100 } \\
\text { g) }\end{array}$ & 103.02 & 52.93 & 72.28 & 78.22 & 73.64 & 59.09 & 112.09 & 75.62 & 88.55 \\
\hline $\begin{array}{l}\text { density } \\
(\mathrm{g} / \mathrm{cm} 3)\end{array}$ & 1.34 & 1.29 & 0.87 & 1.30 & 1.75 & 0.87 & 1.04 & 1.59 & 1.59 \\
\hline prosity (\%) & 2.01 & 3.69 & 2.63 & 6.30 & 9.94 & 2.79 & 4.06 & 6.20 & 5.64 \\
\hline
\end{tabular}


NO.10 Journal of Petroleum Research \& Studies ( JPR \& S)

\begin{tabular}{|c|c|c|c|c|c|c|c|c|c|}
\hline $\begin{array}{l}\mathrm{Ca} \\
(\mathrm{mol} / \mathrm{cm} 3)\end{array}$ & 1.65526103 & 2.43472098 & 1.51363811 & 6.08662636 & 12.37473149 & 1.48188801 & 4.00204083 & 6.85341008 & 7.01755881 \\
\hline $\begin{array}{l}\mathrm{Cc} \\
(\mathrm{mol} / \mathrm{cm} 3)\end{array}$ & 0.260988 & 0.597997048 & 0.488635 & 0.697268 & 0.853753 & 0.561679 & 0.439552 & 0.599946 & 0.484694 \\
\hline $\begin{array}{l}\text { Cs } \\
(\mathrm{mol} / \mathrm{cm} 3)\end{array}$ & 0.3270000 & 0.3270000 & 0.3270000 & 0.3270000 & 0.3270000 & 0.3270000 & 0.3270000 & 0.3270000 & 0.3270000 \\
\hline Ks & 5.061960 & 7.445630 & 4.628863 & 18.613536 & 37.843216 & 4.531768 & 12.238657 & 20.958441 & 21.460424 \\
\hline R ca-w & 1.63 & 1.63 & 1.63 & 1.63 & 1.63 & 1.63 & 1.63 & 1.63 & 1.63 \\
\hline$\sigma$ & 0.7697 & 0.7295 & 0.7051 & 0.7975 & 0.8374 & 0.6915 & 0.8011 & 0.8194 & 0.8374 \\
\hline
\end{tabular}

Table (2) The Specification of Fluids

\begin{tabular}{|l|l|l|}
\hline Specifications & Pore Fluid & Drilling Fluid \\
\hline TDS (mg/l) & 57368 & 197252 \\
\hline Total mobility (mol/kg) & 1963 & 6750 \\
\hline Total molar mass (g/mol) & 58.4 & 58.4 \\
\hline The calculated ionic strength & 1.10 & \\
\hline $\begin{array}{l}\text { Activity coefficient for } \\
\text { monovalent ions }\end{array}$ & 0.555 & \\
\hline $\begin{array}{l}\text { Activity coefficient for } \\
\text { divalent ions }\end{array}$ & 0.0947 & 152.7 \\
\hline Osmotic pressure (bar) & 44.4 & \\
\hline
\end{tabular}




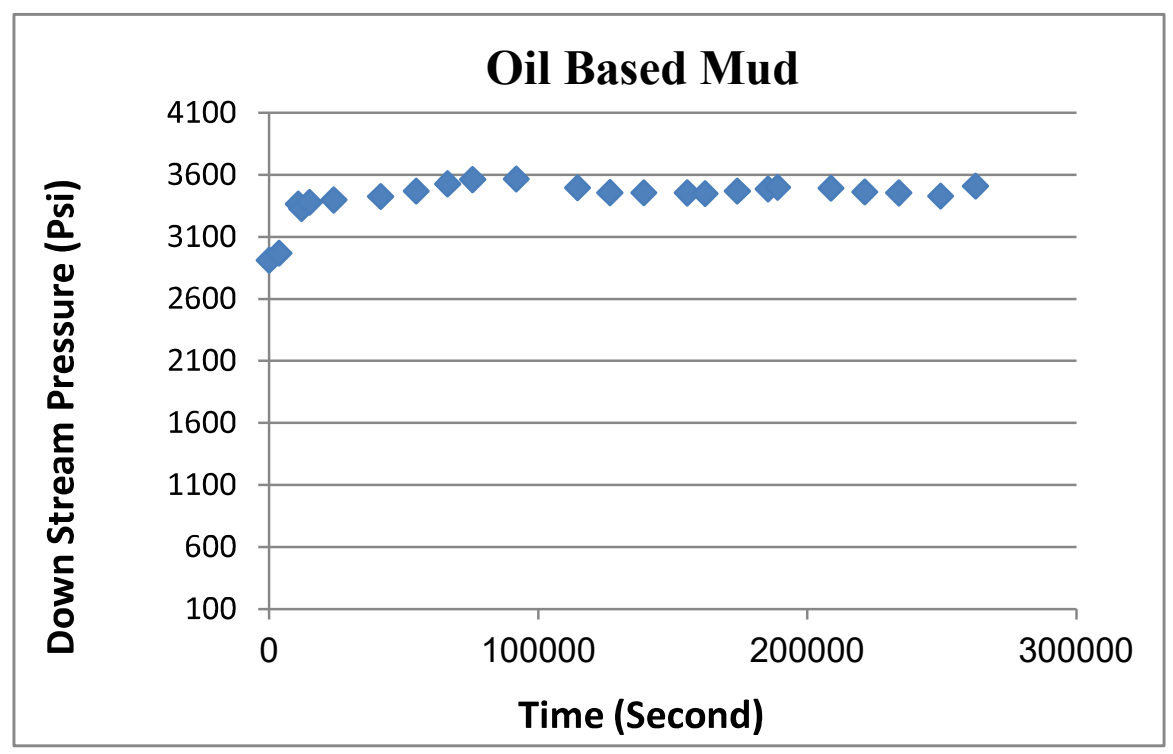

Fig. (1) Downstream pressure changes in core sample used by oil based mud

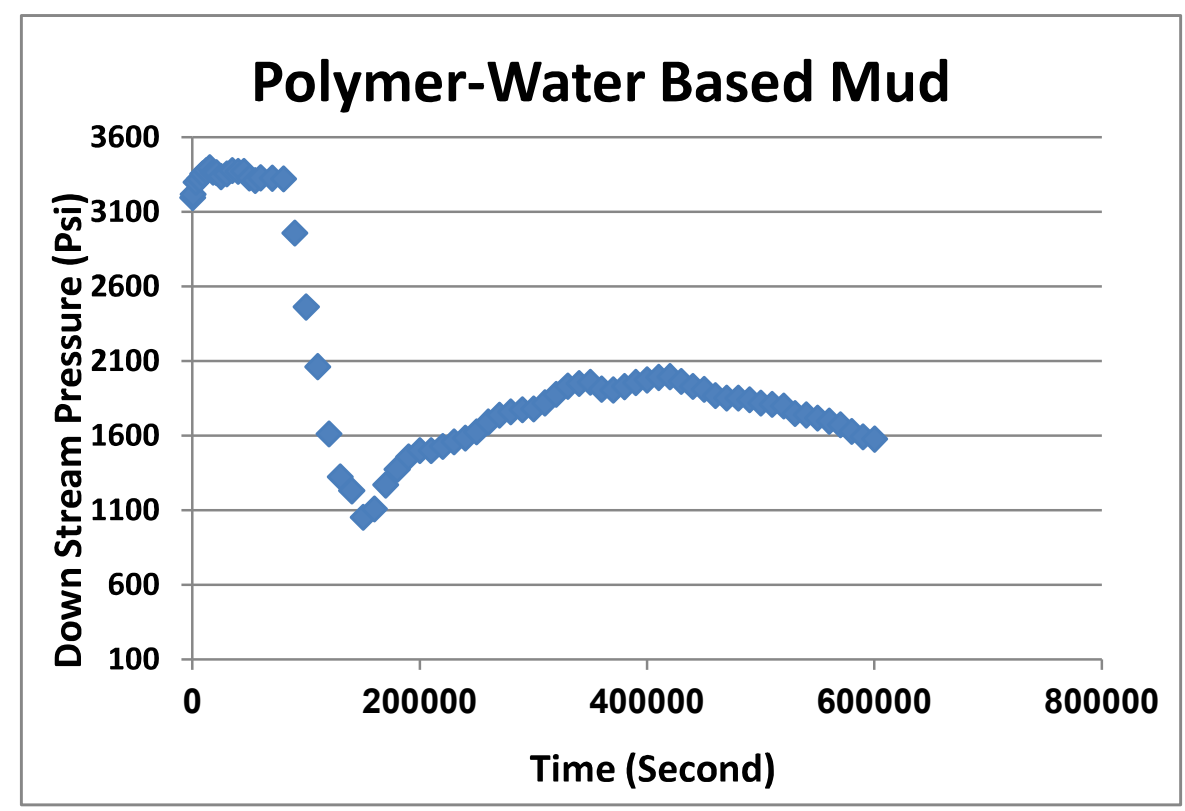

Fig. (2) Downstream pressure changes in core sample used by water-polymer based mud 
NO.10 Journal of Petroleum Research \& Studies ( JPR \& S)

\section{$\underline{\text { Refrences }}$}

1. Van Oort E., Hale A.H., Mody F.K, SPE Annual Conference and Exhibition held in New Orleans, LA, SPE 28309, Sept. 25-28. , (1994).

2. Hilary I. Inyang, Sunyoung Bae, Chemosphere 58, 19-31, (2005).

3. Van Oort E, Journal of Petroleum Science and Engineering, 38, 213235, (2008).

4. Jiankang G.,Jeinian Y.,Hongjing Z., Journal of Petroleum Science and Engineering, 50,195-203 , (2006).

5. Khodja M., Canselier J.P., Bergaya F., Fourar K., Khodja M., Applied Clay Science, 49, 383-393, 2010.

6. Yuanzhi Q., Xiaoqing L., Laifang Z., Yi'nao S, Applied Clay Science 44 265-268, (2009).

7. Van Oort E., Hale A.H., Mody F.K, Baroid Drilling Fluids Inc. SPE 30499, (1995).

8. Marine I.W., Fritz S. J, Water Resources Res. 17, 73-82, (1981).

9. Chee P. Tan, Brain G. Richards, SPE 36971, Asia Pacefic Oil and Gas Conference in Adelaide, (1996). 